\title{
Phase constraint for the waves diffracted by lossless symmetrical gratings at Littrow mount
}

\author{
Cristiano M. B. Cordeiro, Edson J. de Carvalho, and Lucila Cescato \\ Physics Institute “Gleb Wataghin”-University of Campinas, 13083-970, Campinas, Brazil \\ Agnaldo A. Freschi \\ Department of Physics, University of the São Paulo State, 13500-970, Rio Claro, Brazil
}

Lifeng Li

Department of Precision Instruments, Tsinghua University, Beijing 100084, China

Received April 4, 2005; accepted April 22, 2005

\begin{abstract}
The energy conservation of grating diffraction is analyzed in a particular condition of incidence in which two incident waves reach a symmetrical grating from the two sides of the grating normal at the first-order Littrow mounting. In such a situation the incident waves generate an interference pattern with the same period as the grating. Thus in each direction of diffraction, interference occurs between two consecutive diffractive orders of the symmetrical incident waves. By applying only energy conservation and the geometrical symmetry of the grating profile to this problem it is possible to establish a general constraint for the phases and amplitudes of the diffracted orders of the same incident wave. Experimental and theoretical results are presented confirming the obtained relations. (C) 2006 Optical Society of America

OCIS codes: $050.1950,050.5080$.
\end{abstract}

\section{INTRODUCTION}

In the past forty years different methods have been successfully employed to solve the diffraction problem of surface relief gratings. The main purpose of these theories has been the calculation of the diffraction efficiencies as a function of the grating parameters, such as depth, period, and shape of the profile. In recent years, however, many applications of surface relief gratings in resonant and subwavelength domains as polarizing elements have been developed. For the design of such elements the phase of the diffracted waves plays a crucial role. ${ }^{1,2}$ Most theories allow the calculation of such phases; however, the absolute phase values have no physical meaning, and only the relative phase differences are experimentally measurable. In order to check the theoretical phase calculations, reciprocity and energy conservation were used to establish phase constraints for some particular cases involving only four diffracted orders. ${ }^{3,4}$

In this paper we obtain a general constraint for the phases of the diffracted waves by applying only energy conservation and geometric symmetry for a lossless grating at symmetrical Littrow mounting. Experimental and theoretical results are presented confirming the obtained relations.

\section{WAVE MIXING CONDITION OR SYMMETRICAL LITTROW MOUNTING}

If a symmetrical grating (of period $\Lambda$ ) is illuminated simultaneously and symmetrically by two coherent waves (fields $\mathbf{E}_{r}$ and $\mathbf{E}_{s}$ ) of the same wavelength and at the first- order Littrow mounting ( $\theta=-\theta_{L}$ and $\theta_{L}$, respectively), then from the grating equation, in the $i$ th direction of diffraction there is a superimposition of two diffracted fields, $\mathbf{E}_{r(i)}$, and $\mathbf{E}_{s(i-1)}$. Such a situation is illustrated in Fig. 1 for the transmitted orders (the reflected orders are omitted for simplicity). The total number of existing transmitted orders $(2 N)$ and reflected orders $(2 M)$ depends on the wavelength-to-grating period $(\Lambda)$ ratio and on the refractive index of the grating material.

This sum or superimposition is also called wave mixing, ${ }^{5}$ and the resulting irradiance in the $i$ th diffraction direction is given by

$$
I_{i}=C\left|\mathbf{E}_{i}\right|^{2}=I_{r(i)}+I_{s(i-1)}+2 \sqrt{I_{r(i)} I_{s(i-1)}} \cos \left(\psi+\varphi_{r(i)}-\varphi_{s(i-1)}\right),
$$

with $C$ being a constant that depends on the refractive index of the media and $\mathbf{E}_{i}$ the sum of the two diffracted fields $\mathbf{E}_{r(i)}$, and $\mathbf{E}_{s(i-1)} . \psi$ is the phase difference between the incident waves, which represents the phase shift between the grating itself and the interference pattern generated by the two incident waves $\mathbf{E}_{r}$ and $\mathbf{E}_{s} . \varphi$ is the phase of each diffracted wave, caused by diffraction. $I_{r(i)}$ and $I_{s(i-1)}$ are the irradiances of the diffracted waves $\mathbf{E}_{r(i)}$ and $\mathbf{E}_{s(i-1)}$, and $I_{r}$ and $I_{s}$ are the irradiances of the incident waves $\mathbf{E}_{r}$ and $\mathbf{E}_{s}$, respectively.

If the grating is lossless, the sum of the irradiances $I_{i}$ in all the existing diffraction directions (reflected and transmitted) must be equal to the sum of the irradiances of the incident waves $I_{r}+I_{s}$. Using the superscripts $T$ for the 


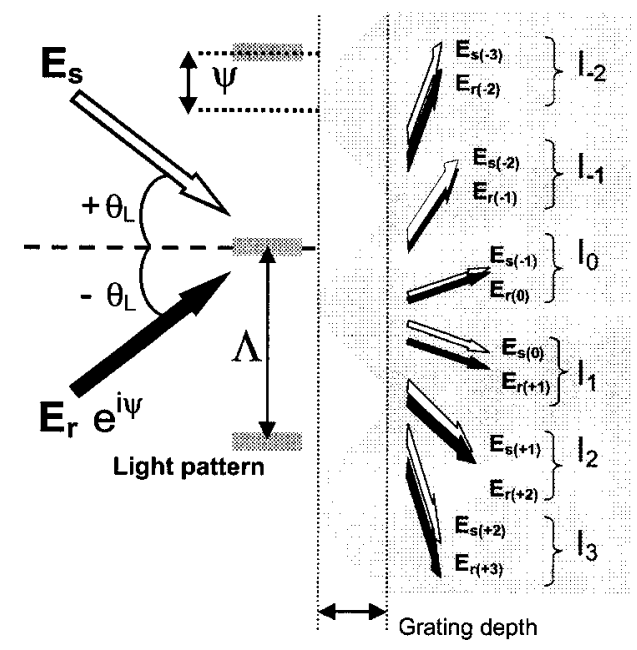

Fig. 1. Interference scheme between the transmitted diffracted orders at symmetrical Littrow mounting. The reflected diffracted orders are omitted for simplicity.

transmitted orders and $R$ for the reflected orders, this energy conservation can be written as

$$
\begin{aligned}
& \sum_{i=-N+1}^{N}\left[I_{r(i)}^{T}+I_{s(i-1)}^{T}+\sqrt{I_{r(i)}^{T} I_{s(i-1)}^{T}} \cos \left(\psi+\varphi_{r(i)}^{T}-\varphi_{s(i-1)}^{T}\right)\right] \\
& \quad+\sum_{i=-M+1}^{M}\left[I_{r(i)}^{R}+I_{s(i-1)}^{R}+\sqrt{I_{r(i)}^{R} I_{s(i-1)}^{R}} \cos \left(\psi+\varphi_{r(i)}^{R}-\varphi_{s(i-1)}^{R}\right)\right] \\
& \quad=I_{r}+I_{s} .
\end{aligned}
$$

The irradiances of the diffracted waves can be written in terms of their diffraction efficiencies $\eta$ as

$$
\begin{aligned}
& I_{r(i)}^{R}=\eta_{r(i)}^{R} I_{r}, \\
& I_{r(i)}^{T}=\eta_{r(i)}^{T} I_{r}, \\
& I_{s(i)}^{R}=\eta_{s(i)}^{R} I_{s}, \\
& I_{s(i)}^{T}=\eta_{s(i)}^{T} I_{s} .
\end{aligned}
$$

By using the fact that for a lossless grating, energy conservation must be valid for each individual incident wave $\mathbf{E}_{s}$ and $\mathbf{E}_{r}$, we have

$$
\begin{gathered}
\sum_{i=-N+1}^{N} \eta_{s(i-1)}^{T}+\sum_{i=-M+1}^{M} \eta_{s(i-1)}^{R}=1 \\
\sum_{i=-N+1}^{N} \eta_{r(i)}^{T}+\sum_{i=-M+1}^{M} \eta_{r(i-1)}^{R}=1 .
\end{gathered}
$$

Substituting Eqs. (3)-(8) into Eq. (2), it is possible to obtain the following general constraint relating the phases and the diffraction efficiencies of all existing diffraction orders of a lossless grating at Littrow mounting:

$$
\begin{aligned}
& \sum_{i=-M+1}^{M} \sqrt{\eta_{r(i)}^{R} \eta_{s(i-1)}^{R}} \cos \left(\psi+\varphi_{r(i)}^{R}-\varphi_{s(i-1)}^{R}\right) \\
& \quad+\sum_{i=-N+1}^{N} \sqrt{\eta_{r(i)}^{T} \eta_{s(i-1)}^{T}} \cos \left(\psi+\varphi_{r(i)}^{T}-\varphi_{s(i-1)}^{T}\right)=0 .
\end{aligned}
$$

If the grating presents a symmetrical profile (in relation to the bisector of the incident beams), the diffraction efficiencies and the phases of the symmetrical $\mathbf{E}_{r}$ and $\mathbf{E}_{s}$ incident beams must be equal:

$$
\begin{aligned}
& \varphi_{r(i)}=\varphi_{s(-i)}, \\
& \eta_{r(i)}=\eta_{s(-i)} .
\end{aligned}
$$

Thus the constraint relation expressed by Eq. (9) simplifies to

$$
\begin{aligned}
& \sum_{i=1}^{M} \sqrt{\eta_{s(-i)}^{R} \eta_{s(i-1)}^{R}} \cos \left(\varphi_{s(-i)}^{R}-\varphi_{s(i-1)}^{R}\right) \\
& \quad+\sum_{i=1}^{N} \sqrt{\eta_{s(-i)}^{T} \eta_{s(i-1)}^{T}} \cos \left(\varphi_{s(-i)}^{T}-\varphi_{s(i-1)}^{T}\right)=0 .
\end{aligned}
$$

This general constraint relates the phases and the amplitudes of all existing diffracted orders of the same incident $\mathbf{E}_{s}$ wave $\left(\theta=+\theta_{L}\right)$ for a lossless symmetrical grating. A corresponding result can be obtained for the $\mathbf{E}_{r}\left(\theta=-\theta_{L}\right)$ incident wave. This constraint can be used to check the phase and amplitude values of the diffracted waves calculated by any theory.

Although the same constraint expressed by Eq. (12) can be derived in grating theory from the unity of the scattering matrix for a lossless grating, ${ }^{6}$ the above derivation gives a better physical insight into such phases. The analysis of the distribution of the energy between the diffracted orders allows, for example, previewing the phase difference behavior as a function of the geometrical parameters of the grating.

Applying the constraint relation to a grating whose period is small enough to allow just four diffracted orders (the minus first and the zeroth diffracted orders by transmission and by reflection) produces

$$
\sqrt{\eta_{-1}^{R} \eta_{0}^{R}} \cos \left(\varphi_{-1}^{R}-\varphi_{0}^{R}\right)+\sqrt{\eta_{-1}^{T} \eta_{0}^{T}} \cos \left(\varphi_{-1}^{T}-\varphi_{0}^{T}\right)=0
$$

This relation represents the same phase constraint obtained by Botten ${ }^{3}$ and Botten et al. ${ }^{4}$ by using the principle of reciprocity, energy conservation, and the symmetry properties of lossless diffraction gratings.

If the grating is a perfectly conducting grating (totally reflecting grating), the efficiencies of the transmitted orders are null; thus

$$
\varphi_{-1}^{R}-\varphi_{0}^{R}=m+\pi / 2,
$$

with $m$ being an integer.

The same occurs if the diffraction efficiencies of the reflected orders are negligible, as for example in the case of a volume grating (Bragg grating). In this case, 


$$
\varphi_{-1}^{T}-\varphi_{0}^{T}=m+\pi / 2 \text {. }
$$

This $\pi / 2$ value of the phase difference between the first and zeroth diffracted orders is a well-known result from the coupled wave theory ${ }^{7}$ for dielectric volume gratings. The same $\pi / 2$ phase value appears in the scalar diffraction theory ${ }^{8}$ as the phase difference between successive orders diffracted by phase gratings.

From the above results we can expect that the phase difference between the first and zeroth orders deviates from the $\pi / 2$ value if the diffraction efficiencies of the remaining diffracted orders are not negligible.

\section{THEORETICAL RESULTS}

The theoretical phases and amplitudes of diffracted waves were calculated for gratings of three different periods $(\Lambda=0.4,0.6$, and $0.8 \mu \mathrm{m})$ as a function of the grating depth by using the coordinate transformation method (the $\mathrm{C}$ method). ${ }^{9} \mathrm{~A}$ sinusoidal relief grating at Littrow mounting was assumed with TE polarization and wavelength $\lambda=457.9 \mathrm{~nm}$. For the dielectric material of the relief grating we consider a real refractive index $n=1.645$.

Table 1 shows the results of the diffraction efficiencies and phases of all the existing diffracted orders as well as the values of both product terms in Eq. (12) for a grating of period $0.4 \mu \mathrm{m}$ and depth $0.2 \mu \mathrm{m}$. For this grating there are only two transmitted diffracted orders and two reflected diffracted orders; thus both products must be equal with opposite sign. Table 2 shows the corresponding results for a grating of period $0.6 \mu \mathrm{m}$ and depth $0.5 \mu \mathrm{m}$. For this grating there are four transmitted diffracted orders and two reflected diffracted orders; thus the sum of the three product terms must equal zero. Table 3 shows the results for a grating of period $0.8 \mu \mathrm{m}$ and depth $0.6 \mu \mathrm{m}$. For this grating there are six transmitted diffracted orders and four reflected diffracted orders; thus the sum of the five product terms must equal zero.

Note that, independent of the period (number of orders) and depth of the grating, the sum of the products remains near zero, confirming the validity of the constraint stated in Eq. (12) and that both amplitude and phase calculated by the employed method ${ }^{9}$ are credible.

Figure 2 shows a graph of the phase difference between the minus first diffracted order and the zeroth diffracted order by transmission $\left(\varphi_{-1}^{T}-\varphi_{0}^{T}\right)$ for sinusoidal surface re-

\section{Table 1. Diffraction Efficiencies, Phases, and Product Terms for a Sinusoidal Grating of Period $0.4 \mu \mathrm{m}$ and Depth $0.2 \mu \mathrm{m}$}

\begin{tabular}{ccc}
\hline Order & $\eta$ & $\varphi\left(^{\circ}\right)$ \\
\hline$-1 \mathrm{~T}$ & 0.18378 & -159.223 \\
$\mathrm{OT}$ & 0.78592 & -67.400 \\
$-1 \mathrm{R}$ & 0.01378 & +69.755 \\
$0 \mathrm{R}$ & 0.01652 & +33.020 \\
\hline$\sqrt{\eta_{-1}^{R} \eta_{0}^{R}} \cos \left(\varphi_{-1}^{R}-\varphi_{0}^{R}\right)$ & $\sqrt{\eta_{-1}^{T} \eta_{0}^{T}} \cos \left(\varphi_{-1}^{T}-\varphi_{0}^{T}\right)$ \\
\hline \multicolumn{2}{c}{$0.01209^{a}$} & $-0.01209^{a}$ \\
\hline
\end{tabular}

${ }^{a}$ Value of product terms in Eq. (13) calculated using data from upper part of table.
Table 2. Diffraction Efficiencies, Phases, and

Product Terms for a Sinusoidal Grating of Period $0.6 \mu \mathrm{m}$ and Depth $0.5 \mu \mathrm{m}$

\begin{tabular}{|c|c|c|}
\hline Order & $\eta$ & $\varphi\left(^{\circ}\right)$ \\
\hline $2 \mathrm{~T}$ & 0.12465 & -114.561 \\
\hline$-1 \mathrm{~T}$ & 0.33247 & 101.015 \\
\hline $0 \mathrm{~T}$ & 0.20777 & 146.126 \\
\hline $1 \mathrm{~T}$ & 0.31934 & 81.953 \\
\hline$-1 R$ & 0.00583 & 135.301 \\
\hline $0 \mathrm{R}$ & 0.00994 & 94.930 \\
\hline$\sqrt{\eta_{-1}^{R} \eta_{0}^{R}} \cos \left(\varphi_{-1}^{R}-\varphi_{0}^{R}\right)$ & $\sqrt{\eta_{-1}^{T} \eta_{0}^{T}} \cos \left(\varphi_{-1}^{T}-\varphi_{0}^{T}\right)$ & $\sqrt{\eta_{-2}^{T} \eta_{1}^{T}} \cos \left(\varphi_{-2}^{T}-\varphi_{1}^{T}\right)$ \\
\hline $0.00580^{a}$ & $0.18549^{a}$ & $-0.19129^{a}$ \\
\hline
\end{tabular}

${ }^{a}$ Value of product terms in Eq. (12) calculated using data from upper part of table.

Table 3. Diffraction Efficiencies, Phases, and Product Terms for a Sinusoidal Grating of Period $0.8 \mu \mathrm{m}$ and Depth $0.6 \mu \mathrm{m}$

\begin{tabular}{rcr}
\hline Order & $\eta$ & \multicolumn{1}{c}{$\varphi\left(^{\circ}\right)$} \\
\hline$-3 \mathrm{~T}$ & 0.03598 & -12.010 \\
$-2 \mathrm{~T}$ & 0.10664 & -108.241 \\
$-1 \mathrm{~T}$ & 0.14687 & 75.474 \\
$0 \mathrm{~T}$ & 0.06330 & 87.029 \\
$1 \mathrm{~T}$ & 0.51361 & -6.953 \\
$2 \mathrm{~T}$ & 0.11579 & -160.566 \\
$-2 \mathrm{R}$ & 0.00606 & -40.757 \\
$-1 \mathrm{R}$ & 0.00079 & -43.394 \\
$0 \mathrm{R}$ & 0.00230 & +9.918 \\
$1 \mathrm{R}$ & 0.00868 & -80.105 \\
\hline$\sqrt{\eta_{-3}^{T} \eta_{2}^{T}} \cos \left(\varphi_{-3}^{T}-\varphi_{2}^{T}\right)$ & $\sqrt{\eta_{-2}^{T} \eta_{1}^{T}} \cos \left(\varphi_{-2}^{T}-\varphi_{1}^{T}\right)$ & $\sqrt{\eta_{-1}^{T} \eta_{0}^{T}} \cos \left(\varphi_{-1}^{T}-\varphi_{0}^{T}\right)$ \\
\hline$-0.05507^{a}$ & -0.04581 & 0.09446 \\
\hline$\sqrt{\eta_{-2}^{R} \eta_{1}^{R}} \cos \left(\varphi_{-2}^{R}-\varphi_{1}^{R}\right)$ & $\sqrt{\eta_{-1}^{R} \eta_{0}^{R}} \cos \left(\varphi_{-1}^{R}-\varphi_{0}^{R}\right)$ & \\
\hline 0.00561 & 0.00081 & \\
\hline
\end{tabular}

${ }^{a}$ Value of product terms in Eq. (12) calculated using data from upper part of table.

lief gratings with three different periods $(0.4,0.6$, and $0.8 \mu \mathrm{m})$ as a function of the grating depth. Note that for the grating period of $0.4 \mu \mathrm{m}$ the phase difference remains close to the $\pi / 2$ value, as expected from Eq. (15). In the same figure is shown (right axis) a graph of the sum of the efficiencies of all remaining diffracted orders (excluding the minus first and zeroth orders) as a function of the grating depth. We can observe that the deviation of the phase difference $\left(\varphi_{-1}^{T}-\varphi_{0}^{T}\right)$ from the $\pi / 2$ value with the grating depth starts when the sum of the diffraction efficiencies of the remaining diffracted orders increases, and it occurs in the same sequence of grating period.

\section{EXPERIMENTAL RESULTS}

Using a method proposed in a previous paper, ${ }^{10}$ we measured the phase difference between the minus first and the zeroth transmitted diffracted orders of surface relief gratings at Littrow mounting. During the measurement a 
grating recorded in a photoresist film of AZ 1518, coated on a glass substrate, is repositioned in the same setup in which it was recorded. ${ }^{10}$ In order to avoid the effects of the reflection at the rear side of the substrate, the glass substrate was index matched with a glass prism.

The accuracy of the measurement is dependent on the accomplishment of a high-precision repositioning, when a moiré-like pattern should be formed. ${ }^{10}$ The experimental measurements of the phase difference between the nega- tive first and the zeroth transmitted diffracted orders $\left(\varphi_{-1}^{T}-\varphi_{0}^{T}\right)$ for holographic surface relief gratings of period $0.8 \mu \mathrm{m}$ and different depths are shown in Fig. 3. For comparison, in the same figure are shown the theoretical expected curves for phase difference $\left(\varphi_{-1}^{T}-\varphi_{0}^{T}\right)$ for three different grating profiles: sinusoidal, lamellar, and triangular. Note that despite the large experimental errors, the experimental measurements of the phase difference $\left(\varphi_{-1}^{T}-\varphi_{0}^{T}\right)$ follow the expected theoretical curve for

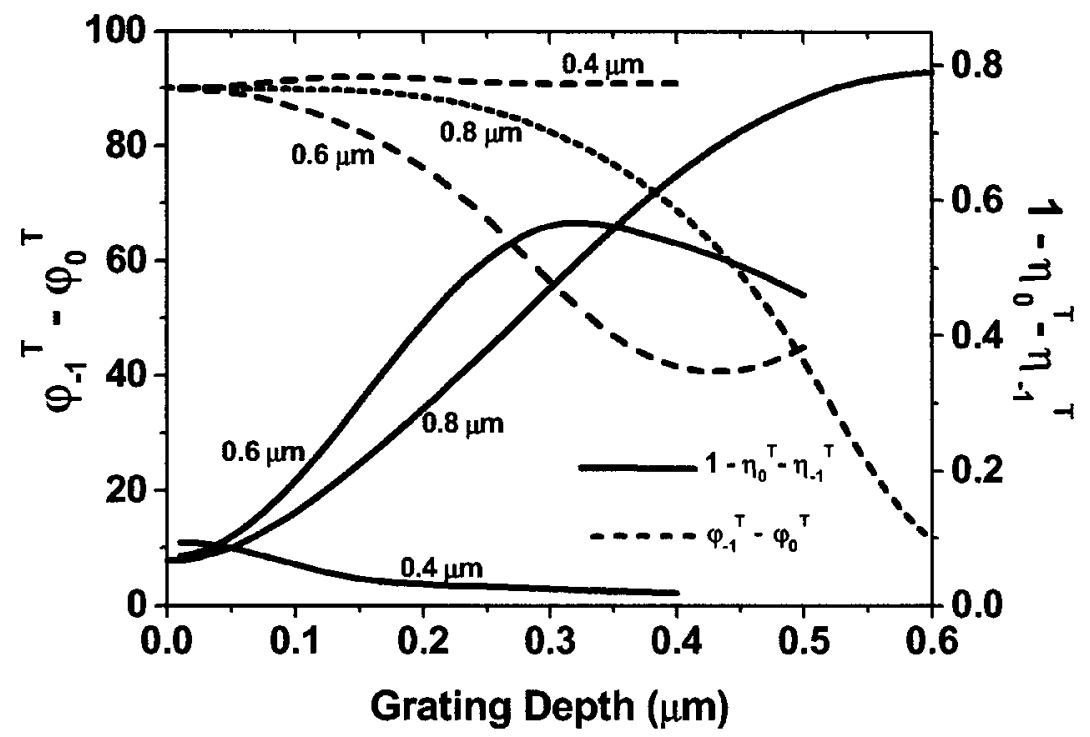

Fig. 2. Phase difference between the minus first and the zeroth diffracted orders by transmission $\left(\varphi_{-1}^{T}-\varphi_{0}^{T}\right)$ as a function of the grating depth for sinusoidal relief gratings in photoresist $(n=1.645)$ for the TE polarization and $\lambda=457.9 \mathrm{~nm}$ and for three different grating periods $0.4,0.6$, and $0.8 \mu \mathrm{m}$. In the same graphic (right scale) is shown the sum of the diffraction efficiencies of all other existing diffracted orders (excluding the minus first and zeroth).

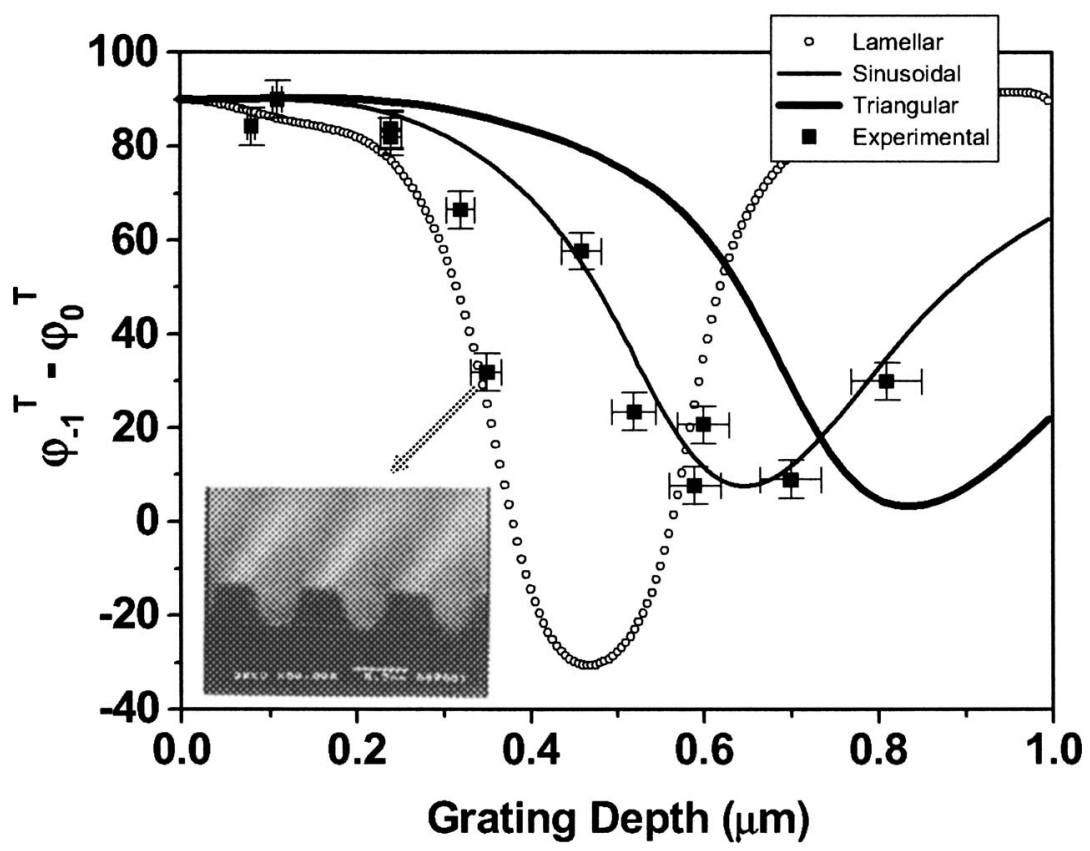

Fig. 3. Experimental results for the phase difference between the minus first and zeroth transmitted diffracted orders $\left(\varphi_{-1}^{T}-\varphi_{0}^{T}\right)$ for a holographic surface relief photoresist grating of period $0.8 \mu \mathrm{m}$ with different grating depth. In the same figure are shown the theoretical phase difference between the first and zeroth transmitted diffracted orders $\left(\varphi_{-1}^{T}-\varphi_{0}^{T}\right)$ as a function of the grating depth for three different grating profiles: lamellar (with filling factor $=0.5$ ), sinusoidal, and triangular. The inset is the scanning electron micrograph of the cross section of the indicated sample. 


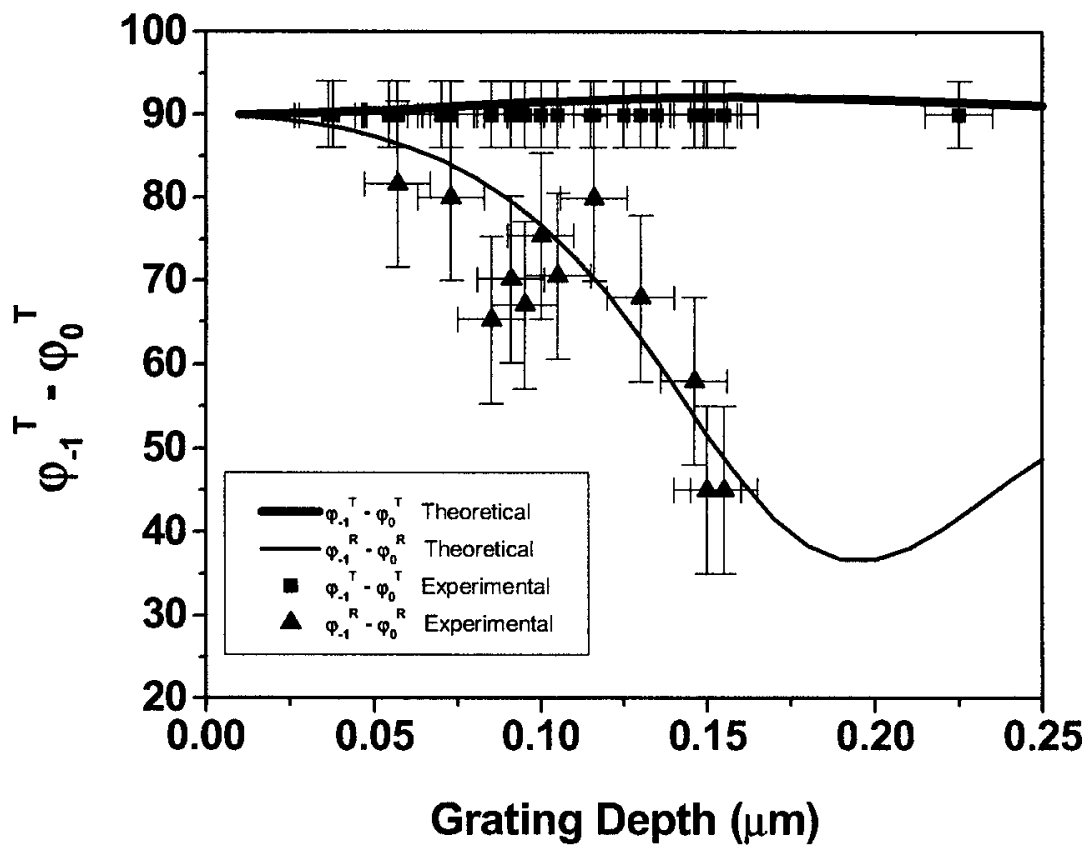

Fig. 4. Experimental measurements and corresponding theoretical curves for the phase difference between the minus first and zeroth diffracted orders $\left(\varphi_{-1}^{T}-\varphi_{0}^{T}\right)$ by transmission and by reflection $\left(\varphi_{-1}^{R}-\varphi_{0}^{R}\right)$ for a holographic surface relief photoresist grating $(n=1.645)$ of period $0.4 \mu \mathrm{m}$ as a function of the grating depth for the TE polarization and $\lambda=457.9 \mathrm{~nm}$.

the sinusoidal profile. Some experimental points deviate from the curve corresponding to the sinusoidal grating profile, approaching the curve corresponding to the lamellar grating profile. An analysis of the grating profiles of such samples by scanning electron microscopy confirm the changes in the sinusoidal profile, as can be seen in the inset photograph.

The departure of the phase difference $\left(\varphi_{-1}^{T}-\varphi_{0}^{T}\right)$ from the $\pi / 2$ value as a function of the grating depth is different for the three different grating profiles. This occurs as a result of the different increase, in energy of the remaining diffraction orders as the grating depth increases, for the different grating profiles. As the sum of the efficiencies of the remaining diffracted orders for a lamellar grating increases more rapidly with the depth than that for a sinusoidal grating profile, the departure from the $\pi / 2$ value also occurs more rapidly. For the same reason the opposite behavior should be expected for the triangularshaped grating.

Figure 4 shows the experimental measurement of the phase difference $\left(\varphi_{-1}^{T}-\varphi_{0}^{T}\right)$ and $\left(\varphi_{-1}^{R}-\varphi_{0}^{R}\right)$ for photoresist gratings of period $0.4 \mu \mathrm{m}$ and different depths. The squares show the measurement for the transmitted orders $\left(\varphi_{-1}^{T}-\varphi_{0}^{T}\right)$, and the triangles show the phase difference $\left(\varphi_{-1}^{R}-\varphi_{0}^{R}\right)$ measured for the reflected orders.

The measurement of the phase differences between the reflected orders presents a larger error bar as well as a larger dispersion compared with the transmitted orders, because the same mismatch in the grating replacement produces a greater distortion in the reflected wavefronts than in the transmitted ones. In addition, the reflection at the photoresist-glass interface introduces an error in the phase measurements that can be neglected for the transmitted orders, because their intensities are higher.

Note that for the transmitted orders the phase difference remains close to $\pi / 2$ while the phase difference be- tween the reflected orders does not. This occurs because for this period there are only two diffracted transmitted orders and two reflected diffracted orders, and the diffraction efficiencies of the reflected orders are negligible in comparison with those of the transmitted orders. Thus from Eq. (15) the phase difference between the two more efficient orders must be close to $\pi / 2$.

\section{CONCLUSIONS}

Using only energy conservation and the symmetry properties of the grating, we derived a general constraint relating the phases and amplitudes of the diffracted orders for a lossless symmetrical grating at Littrow mounting. Although this same constraint can be derived from a general treatment of the diffraction problem by using reciprocity, energy conservation, and the symmetry properties of a grating, this formulation gives a physical insight for the phases allowing one to predict the phasedifference behavior as a function of the grating parameters (such as period, depth, and material).

The numerical verification of this phase constraint [Eq. (12)] allows checking the confidence of the calculated amplitude and phase values of the diffracted orders as well as the method of calculation. The experimental measurements of the phase differences between the minus first and zeroth diffracted orders demonstrate that both the behavior expected by the phase constraint and the theoretical calculation of the phases are correct.

\section{ACKNOWLEDGMENTS}

We acknowledge the financial support of the Fundação de Amparo a Pesquisa do Estado de São Paulo (FAPESP) and Conselho Nacional de Pesquisa (CNPq). 
Corresponding author L. Cescato's e-mail address is cescato@ifi.unicamp.br.

\section{REFERENCES}

1. H. Kikuta, Y. Ohira, and K. Ywata, "Achromatic quarterwave plates using the dispersion of form birefringence," Appl. Opt. 36, 1566-1572 (1997).

2. V. Kettunen and F. Wyrowski, "Reflection mode phase retardation by dielectric gratings," Opt. Commun. 158, 41-44 (1998).

3. L. C. Botten, "A new formalism for transmission gratings," Opt. Acta 25, 481-499 (1978).

4. L. C. Botten, J. L. Adams, R. C. McPhedran, and G. H. Derrick, "Symmetry properties of lossless diffraction gratings," J. Opt. 11, 43-52 (1980).
5. D. L. Staebler and J. J. Amodei, "Coupled-wave analysis of holographic storage in $\mathrm{LiNbO}_{3}$," J. Appl. Phys. 43, 1042-1049 (1972).

6. J. L. Uretsky, "The scattering of plane waves from periodic surfaces," Ann. Phys. 33, 400-427 (1965).

7. H. Kogelnik, "Coupled wave theory for thick hologram gratings," Bell Syst. Tech. J. 48, 2909-2947 (1969).

8. J. W. Goodmam, Introduction to Fourier Optics, 2nd ed. (McGraw-Hill, 1996).

9. L. Li, J. Chandezon, G. Granet, and F.-P. Plumey, "Rigorous and efficient grating-analysis method made easy for optical engineers," Appl. Opt. 38, 304-313 (1999).

10. C. M. B. Cordeiro, L. Cescato, A. Freschi, and L. Li, "Measurement of phase differences between the diffracted orders of deep relief gratings," Opt. Lett. 28, 683-685 (2003). 